\title{
Assessment of van der Waals inclusive density functional theory methods for layered electroactive materials
}

\author{
A. Lozano, ${ }^{1,2}$ B. Escribano, ${ }^{1}$ E. Akhmatskaya, ${ }^{1,3}$ and J. Carrasco ${ }^{2}$ \\ 1) Basque Center for Applied Mathematics, Alameda de Mazarredo 14 (48009) Bilbao, Bizkaia, \\ $\operatorname{Spain}^{\mathrm{a})}$ \\ ${ }^{2)}$ CIC EnergiGUNE, Albert Einstein 48 (01510) Miñano, Álava, Spain \\ ${ }^{3)}$ IKERBASQUE, Basque Foundation for Science, E-48013 Bilbao, Spain
}

Computational-driven materials discovery requires efficient and accurate methods. Density functional theory (DFT) meets these two requirements for many classes of materials. However, DFT-based methods have limitations. One significant shortcoming is the inadequate treatment of weak van der Waals (vdW) interactions, which are crucial for layered materials. Here we assess the performance of various vdW-inclusive DFT approaches for predicting the structure and voltage of layered electroactive materials for Li-ion batteries, considering a set of 20 different compounds. We find that the so-called optB86b-vdW density functional improves the agreement with experimental data, closely followed by the latest generation of dispersion correction methods. These approaches yield average relative errors for the structural parameters smaller than $3 \%$. The average deviations for redox potentials are below $0.15 \mathrm{~V}$. Looking ahead, this study identifies accurate methods for Li-ion vdW bound systems, providing enhanced predictive power to DFT-assisted design for developing new types of electroactive materials in general.

\section{INTRODUCTION}

The computational-driven search for new energy materials based on high-throughput $a b$ initio calculations has gathered momentum during the last decade. ${ }^{1}$ Indeed this strategy has already delivered significant experimentally validated predictions in areas such as energy storage, thermoelectrics, photovoltaics, and hydrogen production, to name but a few (see, e. g., Ref. 2 for a recent review). This, along with the rapidly increasing power of computational resources, have resulted in a flurry of interest in the past few years, and computational prediction is now an important field of materials research. ${ }^{3}$

The first step towards efficient high-throughput $a b$ initio studies is to use computationally affordable methods. This is challenging because there is often a compromise between affordability and the required accuracy to adequately predict the materials properties of interest. Density functional theory (DFT) has managed to balance this plight. ${ }^{2,4}$ However, the standard exchange-correlation functionals used in most routine energy materials studies - usually, generalized-gradientapproximation (GGA) functionals - have various wellknown limitations. ${ }^{5}$ One of the key deficiencies is that these functionals do not properly account for nonlocal electronic correlation effects such as van der Waals (vdW) forces. These forces are important interactions in many materials and are crucial to satisfactorily describe sparse matter. ${ }^{6,7}$ The development of vdW-inclusive DFT methods has therefore been a topic of many strands of research over the years (see, e. g., Refs. 8 and 9). Typically, molecules on metal surfaces, ${ }^{10-18}$ three- and twodimensional solids, ${ }^{19-22}$ and molecular complexes ${ }^{23,24}$

\footnotetext{
a) Electronic mail: alozano@bcamath.org
}

have become the workhorse paradigm for examining the impact of $\mathrm{vdW}$ forces on the binding of weakly interacting systems. A general outcome from these investigations is that the inclusion of $\mathrm{vdW}$ forces to GGA functionals often results in improved binding energies and adsorption distances that are in better agreement with available experimental data.

From a solid-state ionic viewpoint, the vdW-inclusive DFT methods enable the accurate description of sparse electrode materials and, in particular, layered compounds. Layered materials are indeed at the forefront of the next emerging cathodes for $\mathrm{Li}$ - and Na-ion batteries. ${ }^{25,26}$ But just a little attention has being paid to thoroughly analyze the effect of $\mathrm{vdW}$ forces on ion insertion in layered electroactive materials. ${ }^{27-31}$ Interestingly, these studies have revealed that the inclusion of $\mathrm{vdW}$ forces into DFT plays an important role in the structure, energetic stability, redox behavior, and ion diffusion of the studied compounds, resulting in better agreement with experiments. However, the actual impact of $\mathrm{vdW}$ forces on these properties often depend on the specific vdW-inclusive method considered (see, e. g., Ref. 27). This is an uneasy position for vdW-inclusive DFT when applied to layered electroactive materials, and it casts doubt on the use of such techniques in high-throughput studies, which should rely on robust general-purpose calculations. Shedding light on this issue can ultimately facilitate the use of high-throughput ab initio calculations to accelerate the future development of layered electroactive materials. It is therefore very timely and important to assess the performance of a broad variety of $\mathrm{vdW}$ inclusive DFT approaches and identify the most suitable available methods to describe such materials.

In this work, we have considered a diverse set of layered electroactive materials for Li-ion batteries by searching the Materials Project: ${ }^{32} \mathrm{Li}_{0-1} \mathrm{C}_{6}$ and $\mathrm{Li}_{0-x} \mathrm{M}_{y} \mathrm{~A}_{z}(\mathrm{M}=$ $\mathrm{Ti}, \mathrm{V}, \mathrm{Mn}, \mathrm{Cr}, \mathrm{Ni}, \mathrm{Co}, \mathrm{Fe}, \mathrm{Sr}, \mathrm{Nb}, \mathrm{Sn}, \mathrm{Ba}$, and some 
mixtures of them, and $\mathrm{A}=\mathrm{O}, \mathrm{S}, \mathrm{Te}$, and $\left.\mathrm{CO}_{3}\right)$. We focused on compounds where the Li-intercalated and empty structures present the same symmetry and, therefore, no phase transition occurs during the (de)lithiation process. We then assessed the performance of specifically selected vdW-inclusive DFT approaches on these materials. To this end, we compared calculated structural parameters and redox potentials with available experimental data.

In general, vdW-inclusive DFT methods can be classified into two groups: ${ }^{8}$ (i) approaches based on semiempirical corrections typically complemented by a dispersion correction to the Kohn-Sham energy, and (ii) nonlocal correlation density functionals, which directly modify the Kohn-Sham Hamiltonian.

The first generations of the dispersion correction methods, termed DFT-D ${ }^{33}$ and DFT-D $2,{ }^{34}$ used constant values for the $C_{6}$ coefficients of each chemical species. Such approximation does not take into account different oxidation or hybridization states of the same element. Therefore, further improvements to these techniques introduced environment-dependent $C_{6}$ coefficients. The DFT-D3 approach proposed by Grimme et al. ${ }^{35}$ included the environmental dependence of $C_{6}$ coefficients by simply taking into account the number of neighbors each atom has. Alternatively, Tkatchenko and Scheffler ${ }^{36}$ proposed a method which rescaled the $C_{6}$ coefficients by considering reference atomic polarizabilities, reference atomic $C_{6}$ coefficients, and effective atomic volumes obtained from dividing the total electron density of the system between the individual atoms using the Hirshfeld partitioning scheme. We are not considering in our study the Tkatchenko and Scheffler method or any of its variants $^{37,38}$ due to their inability to properly reproduce the cell parameters of bulk $\mathrm{Li}$, a consequence of their overestimation of vdW interactions in metallic systems. ${ }^{27}$ More complex environment-dependent $C_{6}$ corrections involve adjustments of the damping function, which corrects the divergence of $C_{6} / r^{6}$ at short inter-atomic separations. For example, a replacement of the underlying zero-damped dispersion correction of DFT-D3 with a Becke-Johnson damping function (DFT-D3BJ) ${ }^{39}$ results in improved accuracy for the reference data of Meath and co-workers ${ }^{40}$ for 1225 complexes. Similarly, Steinmann et al. ${ }^{41}$ have proposed the $\mathrm{dDsC}$ method, that relies on an extended Tang-Toennies damping function. ${ }^{42}$

In the case of the methods which modify the Hamiltonian, usually referred as van der Waals density functional (vdW-DF), the original proposal by Dion et al. ${ }^{43}$ consisted on the addition of a nonlocal correlation functional to the revPBE ${ }^{44}$ exchange term. Afterwards, an improved revision named vdW-DF2 method was reported, ${ }^{45,46}$ where the nonlocal correlation term is added to a revised version of the PW86 exchange functional (rPW86). ${ }^{45}$ This rPW86 exchange functional was introduced to remedy the tendency of the original vdW-DF to yield too large intermolecular equilibrium distances. Similarly, Klimes el al. ${ }^{47,48}$ proposed alternative optimized exchange functionals within the vdW-
DF scheme, showing improved accuracy for a variety of systems. ${ }^{47,48}$ These optimized exchange functionals named as optPBE, optB88, and optB86b are modified versions of the standard Perdew, Burke and Ernzerhof (PBE) ${ }^{49}$ Becke $88,{ }^{50}$ and Becke $86 b^{51}$ functionals, respectively. An alternative strategy for improvement is the so-called Bayesian error estimation functional (BEEF) which uses the vdW-DF2 nonlocal correlation term and a machine learning trained exchange functional fitted to a reference dataset. ${ }^{52}$ The BEEF approach was explicitly designed to handle a diversity of materials (molecule, bulk solid, and surface chemical bondings).

Here we considered a representative variety of methods within each group and, for comparison, the semilocal Perdew-Burke-Ernzerhof (PBE) exchange-correlation functional. ${ }^{49}$ In the case of dispersion-corrected methods we used the latest generation of the Grimme's approach (D3), ${ }^{35}$ the D3 with the Becke-Johnson damping function (D3BJ), ${ }^{39}$ and the so-called dDsC method proposed by Steinmann et al. ${ }^{41}$. We employed PBE as the underlying exchange-correlation functional for all of them. As for nonlocal density functionals, we used the so-called vdW-DF2 of Lee et al., ${ }^{46}$ the Bayesian error estimation functional (BEEF), ${ }^{52}$ and three optimized versions ${ }^{47,48}$ of the original vdW density functional of Dion et al. ${ }^{43}$ referred to as optPBE-vdW, optB88-vdW, and optB86bvdW herein.

\section{COMPUTATIONAL METHODS}

All calculations were performed using the projector augmented wave method ${ }^{53}$ as implemented in the Vienna ab initio simulation package (VASP). ${ }^{54-56}$ The initial setup for each structure was automatically generated using the pymatgen package. ${ }^{57}$

The valence electrons were described within a planewave basis and an energy cutoff of $520 \mathrm{eV}$, whereas the remaining electrons were kept frozen as core states in the projector-augmented wave method. ${ }^{53}$ We used a $\Gamma$ centered grid for the $\mathbf{k}$-points mesh with a density of at least 1000 (8000) points per atom in the reciprocal space for insulating (metallic) systems. For the materials containing transition metals with localized $3 d$ orbitals, the DFT calculations were supplemented with a Dudarev et $a l .{ }^{58}+\mathrm{U}$ correction $(\mathrm{DFT}+\mathrm{U})$. For each metal, we used the $U$ values provided in the Materials Project, ${ }^{32}$ which were determined with the approach outlined by Wang et al. ${ }^{59,60}$ to fit binary oxides formation energies (V: $3.25 \mathrm{eV}$, Co: $3.32 \mathrm{eV}$, Fe: $5.3 \mathrm{eV}, \mathrm{Ni}: 6.2 \mathrm{eV}, \mathrm{Mn}: 3.9 \mathrm{eV})$. The ions with possible magnetization are initialized with a high-spin value in a ferromagnetic configuration as a computational compromise for carrying a high-throughput study. ${ }^{61}$ The only exception are compounds containing $\mathrm{Co}$ and $\mathrm{Ni}$ species, where we initially set their magnetic moments to low-spin values, as this is known to improve convergence and lead to ground state solutions. ${ }^{27,62}$ In addition, the complete set of VASP input and output 


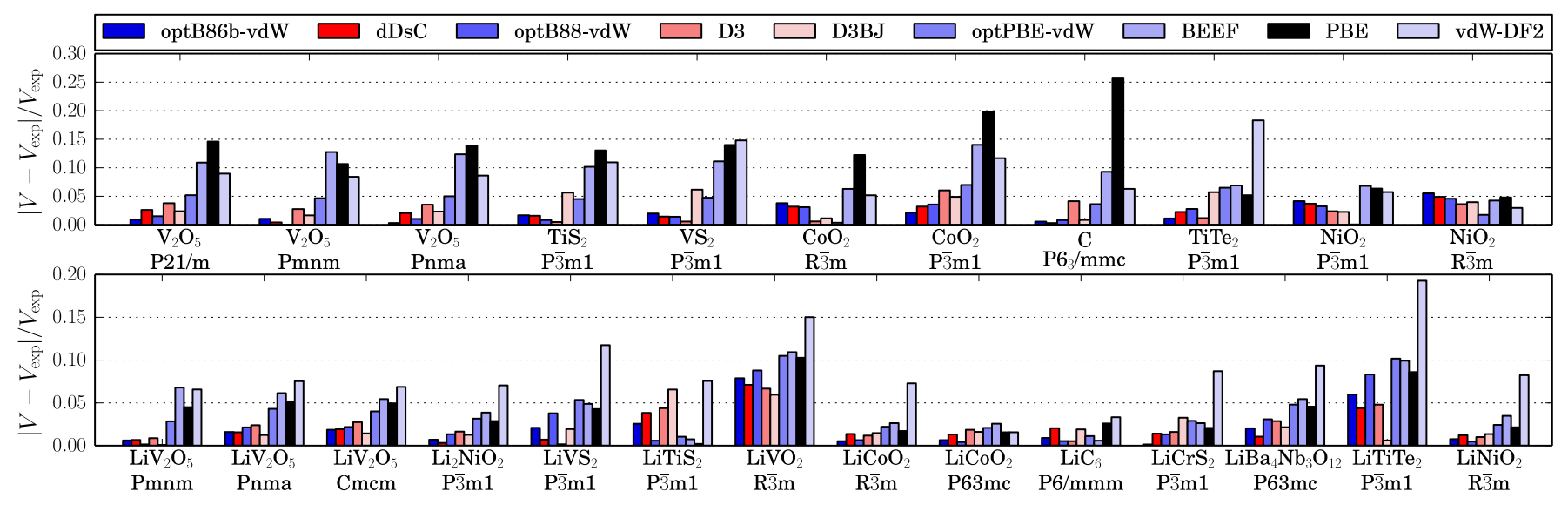

FIG. 1. Relative deviation from the experimental volume $\left(V_{\exp }\right)$ obtained with different vdW-inclusive DFT methods and PBE for a selected set of layered electroactive materials for Li-ion batteries. A blue (red) gradient is used for nonlocal correlation density functional (pairwise dispersion correction) methods, whereas black is used for PBE. The bars order for each system is the same as in the figure legend. Upper panel: empty hosts. Lower panel: Li-intercalated structures.

files can be downloaded from the NoMaD repository via http://nomad-repository.eu, for the details see the Supplementary Information (SI).

\section{RESULTS}

First, we investigated the accuracy of each vdWinclusive method for describing the atomic structure of the nominated compounds. Figure 1 shows the deviation of crystal volumes from experimental data; in Tables S1 and S2 we provide detailed information of the computed and experimental values used to make the figure. In the upper panel of Figure 1 we present the empty hosts, whereas Li-intercalated compounds are shown in the lower panel. Notice that not all of the corresponding lithiated-delithiated pairs are present, since for some cases experimental values are not available. In general, all dispersion-corrected methods based on a pairwise approximation and the optB86b-vdW and optB88$\mathrm{vdW}$ density functionals show the lowest deviations. The mean absolute errors (MAEs) of the computed volumes for these methods are less than $2 \AA^{3}$ (Figure 2), which is equivalent to relative deviations from the experimental values of $3 \%$. In contrast, BEEF, PBE and vdW-DF2 systematically overestimate the volumes by $4-6 \AA^{3}$ (6-9 $\%)$. Interestingly, when comparing the MAEs obtained independently for empty hosts and the corresponding Liintercalated structures (Figure 3), we observed that the behavior of BEEF, PBE and vdW-DF2 is particularly disadvantageous for describing empty hosts, with MAEs larger than $6 \AA^{3}$. This result is somehow expected since the interlayer cohesion of empty hosts is dominated by vdW forces, whereas in Li-intercalated compounds the out-of-plane forces gradually change to electrostatic interactions, which are well described by GGA functionals.

Klimeš et al. ${ }^{48}$ also observed a systematic overestima-

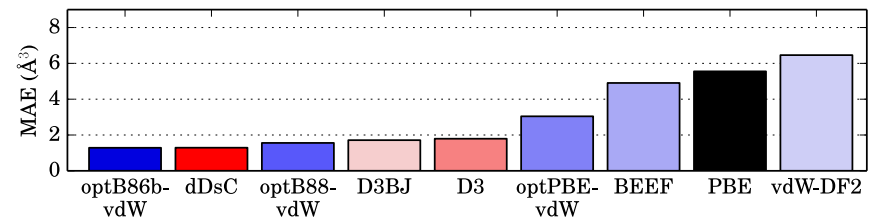

FIG. 2. Mean absolute error relative to experimental values of the predicted volumes for the complete set of compounds shown in Figure 1 using different vdW-inclusive DFT methods and PBE. Same color code as in Figure 1.

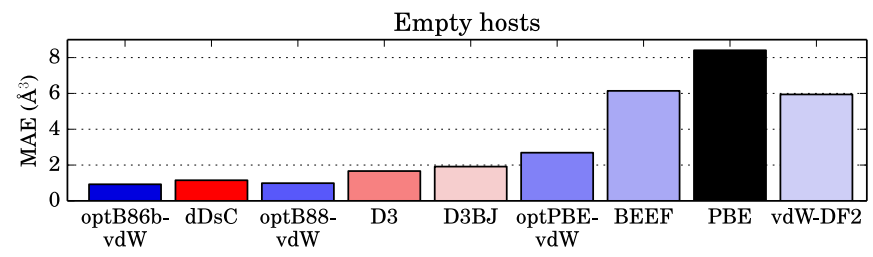
Li-intercalated structures

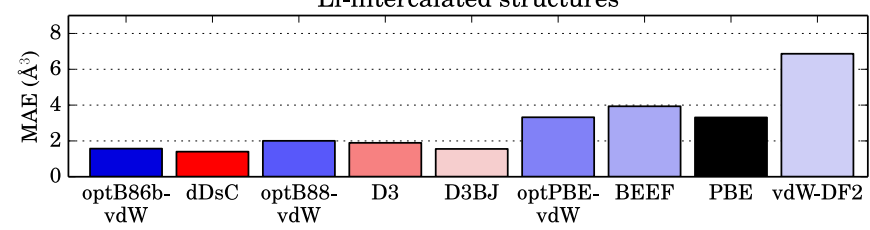

FIG. 3. Mean absolute error relative to experimental values of the predicted volumes for empty hosts (upper panel) and Li-intercalated structures (lower panel) using different vdW-inclusive DFT methods and PBE. Same color code as in Figure 1.

tion of interatomic distances by vdW-DF2 in a variety of bulk solids. The authors linked the failure to the too steep behavior of the exchange enhancement factor $\left(F_{x}\right)$ at low reduced densities. The BEEF functional uses the vdW-DF2 nonlocal correlation term, ${ }^{52}$ which in turn ex- 
plains its poor performance in describing the layered materials considered here.

The development of opt-type exchange functionals was initially aiming to reduce the excessive repulsion at short interatomic distances by improving the $F_{x}$ behavior. In this study, we see that such functionals describe very well indeed the atomic structure of both Li-intercalated and empty hosts, with the optB86b-vdW showing the lowest MAE. Another key finding is that all of the considered dispersion-corrected methods perform equally good, with the dDsC approach showing the lowest MAE within this class of methods and very close to optB86b$\mathrm{vdW}$. It is important to stress that the small deviations found for the investigated opt functionals and dispersioncorrected methods $(\approx 3 \%)$ are comparable to the level of accuracy provided by more computationally expensive random-phase approximation (RPA) calculations. ${ }^{63}$ The RPA method takes into account long-range dynamic correlation effects and is considered as reference for twodimensional materials. ${ }^{64}$ In particular, Björkman et al. ${ }^{65}$ explored a range of layered compounds using RPA and found deviations from the structural experimental values of $\sim 4 \%$.

We discuss now the role of vdW interactions in the average redox potential for $\mathrm{Li}$ insertion relative to a $\mathrm{Li}$ metal anode, $\Phi$, computed as detailed in the SI. Figure 4 shows $\Phi$ for each $\mathrm{Li}_{0 \rightarrow x} \mathrm{M}_{y} \mathrm{~A}_{z}$ pair using $\mathrm{PBE}$ and all considered vdW-inclusive methods (experimental $\Phi$ values are scarce but included when available). A key observation is that, in general, PBE yields lower $\Phi$ values than vdW-inclusive methods and experimental measurements. Similar findings have recently been reported by Aykol et al. ${ }^{27}$ for $\mathrm{LiCoO}_{2}$ polymorphs. This indicates that vdW interactions systematically tend to increase the redox potential. The MAE of each method with respect to the available experimental data is shown in Fig. 5. The deviations vary from 0.11 to $0.28 \mathrm{~V}$, which is translated into errors between 8 to $16 \%$ when considering them relative to the experimental values. Most of the vdW-inclusive methods show smaller MAE values than PBE, with only D3 and vdW-DF2 performing worse. The choice of the vdW-inclusive approach has, therefore, less impact on computed $\Phi$ values than on structural parameters.

It is important to mention that a key aspect for computing accurate redox potentials is a proper description of the localized transition metal $d$ states. But GGA-based DFT calculations have non-canceling self-interaction errors in the energy of such strongly correlated electrons. In particular, these errors can be magnified when the electrons are transferred between very different chemical environments (e. $g$., from the localized $3 d$ state of the transition metal ion to the delocalized $2 s$ state of metallic Li). ${ }^{59}$ In these cases the pragmatic DFT+U approach, in which an effective Hubbard U-like term is added to exchange-correlation functional, has successfully been applied to mitigate the self-interaction error and improve the description of $3 d$ states. ${ }^{66}$ Recently, to avoid the non-obvious choice of the $\mathrm{U}$ value, Seo et al. ${ }^{67}$

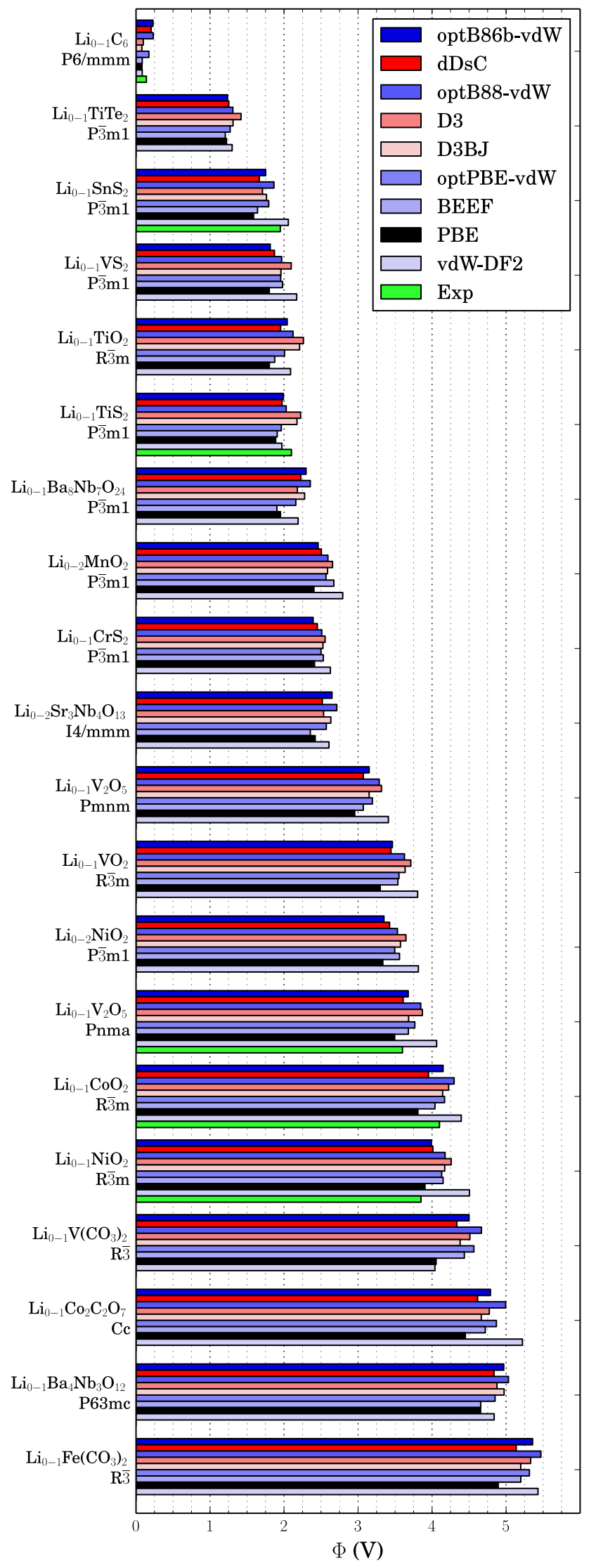

FIG. 4. Computed redox potentials for Li intercalation $(\Phi)$ using different vdW-inclusive DFT methods and PBE. Same color code as in Figure 1. Green bars correspond to experimental values when available. 


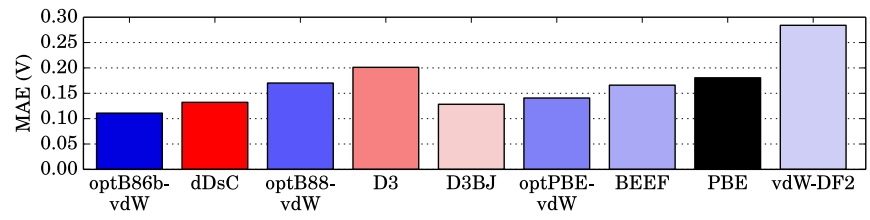

FIG. 5. Mean absolute error relative to experimental values of predicted redox potentials using different vdW-inclusive DFT methods and PBE. Only the systems for which experimental data is available were considered $\left(\mathrm{Li}_{0-1} \mathrm{C}_{6}, \mathrm{Li}_{0-1} \mathrm{SnS}_{2}\right.$, $\mathrm{Li}_{0-1} \mathrm{TiS}_{2}, \mathrm{Li}_{0-1} \mathrm{~V}_{2} \mathrm{O}_{5}, \mathrm{Li}_{0-1} \mathrm{CoO}_{2}$, and $\left.\mathrm{Li}_{0-1} \mathrm{NiO}_{2}\right)$. Same color code as in Figure 1.

proposed the use of hybrid functionals with a fraction of Hartree-Fock exact exchange to improve the prediction of redox potentials. This technique can provide very accurate $\Phi$ values $(\lesssim 2 \%)$, but only after the careful tuning of a mixing parameter for fitting experimental band gaps of the materials under study. Therefore, this kind of approaches are not desirable for high-throughput studies due to their high computational cost and a dependence on experimental information. Here we used the $\mathrm{DFT}+\mathrm{U}$ method with the $\mathrm{U}$ values provided in the Materials Project (MP) ${ }^{32}$ which were derived using the approach outlined by Wang et al. ${ }^{59}$ to fit binary oxides formation energies. Neglecting this correction (i.e. $\mathrm{U}=0.0$ $\mathrm{eV})$ systematically yields too low redox potentials compared with the experimental data, resulting in large MAE values as shown in Figure 6.

The $U$ values provided in the Materials Project and used in this work are optimized for PBE. Arguably, such $\mathrm{U}$ values cannot be blindly transferred to other functionals. Thus, to analyze how the $\mathrm{U}$ correction affects the vdW-inclusive methods, we performed additional calculations on a selection of systems. Specifically, we computed unit cell volumes and redox potentials varying the U parameter (see Figs. S1-3 in SI) using plain PBE, the two vdW-inclusive methods presenting the best performance (optB86b-vdW and $\mathrm{dDsC}$ ) and vdW-DF2, which shows the worst performance according to previous discussions. Essentially, we observed that, regardless of the $\mathrm{U}$ value, the unit cell volumes of empty hosts are always overestimated with PBE. Additionally, when considering a vdW-inclusive approach, the computed redox potential is always higher than that obtained with $\mathrm{PBE}$, and this fact, in general, improves the average result as compared with experimental measurements. Most significantly, the two optimal vdW-inclusive methods (optB86b-vdW and $\mathrm{dDsC}$ ) consistently yield the same trends and results with relative differences of less than $5 \%$ between them. These findings indicate that introducing additional nonlocal electron correlation effects by using a proper vdWinclusive approach can provide even better agreement with experiment than PBE, and the U parameters from the Materials Project show a good transferability in the considered cases.

Finally, it is important to notice that the nature of

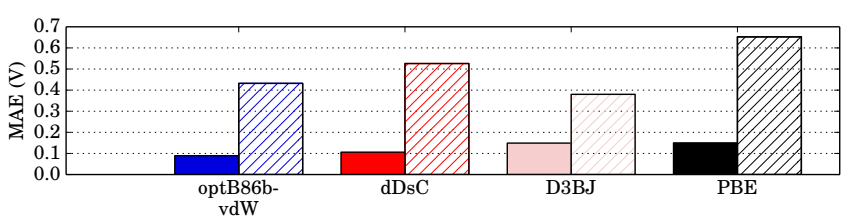

FIG. 6. Mean absolute error relative to experimental values of predicted redox potentials using different vdW-inclusive DFT methods and PBE. Only the systems for which the $+\mathrm{U}$ correction is applicable are included. Full bars correspond to calculations using the $+U$ correction, whereas hatched bars correspond to calculations which neglect this correction $(\mathrm{U}=$ $0.0 \mathrm{eV}$ ).

the excess charge on transition metals upon Li insertion usually depends on the choice of U parameters (see, e.g., Refs. 67, 68 or 69). Typically, an accurate account of band gaps and actual degree of charge localization involves exhaustive studies for each particular system under consideration. Ideally, these evaluations should be carried out considering available experimental data (e.g., valence photoemission spectra) or high-level quantum chemistry benchmark computations (e.g., GW methods). However, such overwhelming task for the 20 redox couples considered here is beyond the scope of this study. Certainly, our DFT $+\mathrm{U}$ results are limited to the U parametrization provided by the Materials Project. In the SI we show the computed projected densities of states for all of the Li-intercalated and empty structures (Figures S4-S7). A thorough U dependence of the electronic structure for each redox couple could be desirable. However, as discussed above, we see that for the assessment of vdW-inclusive methods regarding the properties of interest here (unit cell volume and redox potential), such $\mathrm{U}$ dependence does not change our conclusions.

\section{CONCLUSIONS}

We have assessed a range of recent $\mathrm{vdW}$-inclusive DFT methods (dDsC, D3, D3BJ, optB86b-vdW, optB88-vdW, optPBE-vdW, BEEF, and vdW-DF2) using a diverse set of 20 layered electroactive materials for Li-ion batteries. Our calculations have shown that an accurate treatment of vdW interactions is essential for the accurate prediction of equilibrium atomic structures and average redox potentials. In the case of structural parameters, the inclusion of the vdW interactions is particularly important for the description of delithiated compounds. We found that the performance of BEEF and vdW-DF2 is worse than that of PBE. However, all other methods demonstrate an improved accuracy, presenting deviations of less than $3 \%$ with respect to available experimental data. Overall, optB86b-vdW and dDsC show the best performance for predicting equilibrium geometries. The impact of vdW interactions on the computed average redox potential is less important, with PBE already providing 
values very close to experimental ones. However, we saw a further improvement when explicitly considering vdW interactions through the use of optB86b-vdW, optB88vdW, optPBE-vdW, BEEF, dDsC, or D3BJ. Considering both structural parameters and redox potentials, our analysis indicates that optB86b-vdW achieves the overall best description of the studied layered electroactive materials, closely followed by $\mathrm{dDsC}$ and D3BJ.

In general, the computational evaluation of $\mathrm{vdW}$ inclusive methods, which does not require fitting to experimental data or using computationally expensive techniques, is particularly desirable for designing new energy materials. From such a practical viewpoint, our results are meant to serve as a guidance for the selection of accurate and robust methods for high-throughput DFT schemes.

\section{ACKNOWLEDGMENTS}

B.E. and E.A. acknowledge funding by MINECO (Spain) grant MTM2013-46553-C3-1-P. J.C. acknowledges funding by MINECO grant ENE2016-81020-R and support by The Royal Society through the Newton Alumnus scheme. We are also grateful for computer resources to the Barcelona Supercomputer Center (BSC), i2BASQUE, and the Arina cluster (Universidad del País Vasco/Euskal Herriko Unibertsitatea). This research was supported by the Basque Government through the BERC 2014-2017 program and by the Spanish Ministry of Economy and Competitiveness MINECO: BCAM Severo Ochoa accreditation SEV-2013-0323.

${ }^{1}$ G. Hautier, A. Jain and S. P. Ong, J. Mater. Sci., 2012, 47, $7317-7340$.

${ }^{2}$ A. Jain, Y. Shin and K. A. Persson, Nat. Rev. Mater., 2016, 1, 15004 .

${ }^{3}$ J. Hill, G. Mulholland, K. Persson, R. Seshadri, C. Wolverton and B. Meredig, MRS Bulletin, 2016, 41, 399-409.

${ }^{4}$ J. Hafner, C. Wolverton and G. Ceder, MRS Bull., 2006, 31, 659-668.

${ }^{5}$ K. Burke, J. Chem. Phys., 2012, 136, 150901.

${ }^{6}$ N. Marom, J. Bernstein, J. Garel, A. Tkatchenko, E. Joselevich, L. Kronik and O. Hod, Phys. Rev. Lett., 2010, 105, 046801.

${ }^{7}$ G. Graziano, J. Klimeš, F. Fernandez-Alonso and A. Michaelides, J. Phys.: Condens. Matter, 2012, 24, 424216.

${ }^{8}$ J. Klimeš and A. Michaelides, J. Chem. Phys., 2012, 137, 120901.

${ }^{9}$ S. Grimme, A. Hansen, J. G. Brandenburg and C. Bannwarth, Chem. Rev., 2016, 116, 5105-5154.

${ }^{10}$ N. Atodiresei, V. Caciuc, P. Lazić and S. Blügel, Phys. Rev. Lett., 2009, 102, 136809.

${ }^{11}$ A. K. Kelkkanen, B. I. Lundqvist and J. K. Nørskov, Phys. Rev. B, 2011, 83, 113401.

${ }^{12}$ J. Carrasco, B. Santra, J. Klimeš and A. Michaelides, Phys. Rev. Lett., 2011, 106, 026101.

${ }^{13}$ M. A. Floridia Addato, A. A. Rubert, G. A. Benítez, M. H. Fonticelli, J. Carrasco, P. Carro and R. C. Salvarezza, J. Phys. Chem. C, 2011, 115, 17788-17798.

${ }^{14}$ W. Liu, A. Savara, X. Ren, W. Ludwig, K.-H. Dostert, S. Schauermann, A. Tkatchenko, H.-J. Freund and M. Scheffler, J. Phys. Chem. Lett., 2012, 3, 582-586.

${ }^{15}$ W. Liu, J. Carrasco, B. Santra, A. Michaelides, M. Scheffler and A. Tkatchenko, Phys. Rev. B, 2012, 86, 245405.
${ }^{16}$ H. Yildirim, T. Greber and A. Kara, J. Phys. Chem. C, 2013, 117, 20572-20583.

${ }^{17} \mathrm{~J}$. Carrasco, W. Liu, A. Michaelides and A. Tkatchenko, $J$. Chem. Phys., 2014, 140, 084704.

${ }^{18}$ R. J. Maurer, V. G. Ruiz, J. Camarillo-Cisneros, W. Liu, N. Ferri, K. Reuter and A. Tkatchenko, Prog. Surf. Sci., 2016, 91, $72-$ 100.

${ }^{19}$ T. P. Kaloni, Y. C. Cheng and U. Schwingenschlogl, J. Mater. Chem., 2012, 22, 919-922.

${ }^{20}$ T. Kaloni and U. Schwingenschlögl, Chem. Phys. Lett., 2013, 583, $137-140$.

${ }^{21}$ T. P. Kaloni, G. Schreckenbach and M. S. Freund, J. Phys. Chem. C, 2014, 118, 23361-23367.

${ }^{22}$ A. M. Reilly and A. Tkatchenko, Chem. Sci., 2015, 6, 3289-3301.

${ }^{23}$ A. Tkatchenko, D. Alfè and K. S. Kim, J. Chem. Theory Comput., 2012, 8, 4317-4322.

${ }^{24}$ T. P. Kaloni, G. Schreckenbach and M. S. Freund, Sci Rep, 2016, 6, 36554 .

${ }^{25}$ J. W. Choi and D. Aurbach, Nat. Rev. Mater., 2016, 1, 16013.

${ }^{26}$ M. H. Han, E. Gonzalo, G. Singh and T. Rojo, Energy Environ. Sci., 2015, 8, 81-102.

${ }^{27}$ M. Aykol, S. Kim and C. Wolverton, J. Phys. Chem. C, 2015, 119, 19053-19058.

${ }^{28}$ J. Carrasco, J. Phys. Chem. C, 2014, 118, 19599-19607.

${ }^{29}$ Z. Wang, S. M. Selbach and T. Grande, RSC Adv., 2014, 4, 4069-4079.

${ }^{30}$ S. Thinius, M. M. Islam, P. Heitjans and T. Bredow, J. Phys. Chem. C, 2014, 118, 2273-2280.

${ }^{31}$ V. Riffet, J. Contreras-García, J. Carrasco and M. Calatayud, J. Phys. Chem. C, 2016, 120, 4259-4265.

${ }^{32}$ A. Jain, S. P. Ong, G. Hautier, W. Chen, W. D. Richards, S. Dacek, S. Cholia, D. Gunter, D. Skinner, G. Ceder and K. A. Persson, APL Mater., 2013, 1, 011002.

${ }^{33}$ S. Grimme, J. Comput. Chem., 2004, 25, 1463-1473.

${ }^{34}$ S. Grimme, J. Comput. Chem., 2006, 27, 1787-1799.

${ }^{35}$ S. Grimme, J. Antony, S. Ehrlich and H. Krieg, J. Chem. Phys., 2010, 132, 154104.

${ }^{36}$ A. Tkatchenko and M. Scheffler, Phys. Rev. Lett., 2009, 102, 073005 .

${ }^{37}$ A. Tkatchenko, R. A. DiStasio, R. Car and M. Scheffler, Phys. Rev. Lett., 2012, 108, 236402.

${ }^{38}$ T. Bučko, S. Lebègue, J. G. Ángyán and J. Hafner, J. Chem. Phys., 2014, 141, 034114.

${ }^{39}$ S. Grimme, S. Ehrlich and L. Goerigk, J. Comp. Chem., 2011, 32, 1456-1465.

${ }^{40}$ D. J. Margoliash and W. J. Meath, J. Chem. Phys., 1978, 68, $1426-1431$.

${ }^{41}$ S. N. Steinmann and C. Corminboeuf, J. Chem. Phys., 2011, 134, 044117.

${ }^{42}$ K. T. Tang and J. P. Toennies, J. Chem. Phys, 1984, 80, 37263741.

${ }^{43}$ M. Dion, H. Rydberg, E. Schröder, D. C. Langreth and B. I. Lundqvist, Phys. Rev. Lett., 2004, 92, 246401.

${ }^{44}$ Y. Zhang and W. Yang, Phys. Rev. Lett., 1998, 80, 890-890.

${ }^{45}$ E. D. Murray, K. Lee and D. C. Langreth, J. Chem. Theory Comput., 2009, 5, 2754-2762.

${ }^{46}$ K. Lee, E. D. Murray, L. Kong, B. I. Lundqvist and D. C. Langreth, Phys. Rev. B, 2010, 82, 081101.

${ }^{47}$ J. Klimeš, D. R. Bowler and A. Michaelides, J. Phys.: Condens. Matter, 2010, 22, 022201.

${ }^{48}$ J. Klimeš, D. R. Bowler and A. Michaelides, Phys. Rev. B, 2011, 83, 195131.

${ }^{49}$ J. P. Perdew, K. Burke and M. Ernzerhof, Phys. Rev. Lett., 1996, 77, 3865-3868.

${ }^{50}$ A. D. Becke, Phys. Rev. A, 1988, 38, 3098-3100.

${ }^{51}$ A. D. Becke, J. Chem. Phys., 1986, 85, 7184-7187.

52 J. Wellendorff, K. T. Lundgaard, A. Møgelhøj, V. Petzold, D. D. Landis, J. K. Nørskov, T. Bligaard and K. W. Jacobsen, Phys. Rev. B, 2012, 85, 235149

${ }^{53}$ G. Kresse and D. Joubert, Phys. Rev. B, 1999, 59, 1758-1775. 
${ }^{54}$ G. Kresse and J. Hafner, Phys. Rev. B, 1993, 47, 558-561.

${ }^{55} \mathrm{G}$. Kresse and J. Furthmüller, Comput. Mater. Sci., 1996, 6, 15 -50 .

${ }^{56}$ G. Kresse and J. Furthmüller, Phys. Rev. B, 1996, 54, 11169 11186.

${ }^{57}$ S. P. Ong, W. D. Richards, A. Jain, G. Hautier, M. Kocher, S. Cholia, D. Gunter, V. L. Chevrier, K. A. Persson and G. Ceder, Comp. Mat. Sci., 2013, 68, $314-319$.

${ }^{58}$ S. Dudarev, G. Botton, S. Savrasov, C. Humphreys and A. Sutton, Phys. Rev. B, 1998, 57, 1505-1509.

${ }^{59}$ L. Wang, T. Maxisch and G. Ceder, Phys. Rev. B, 2006, 73, 195107.

${ }^{60}$ G. Hautier, S. P. Ong, A. Jain, C. J. Moore and G. Ceder, Phys. Rev. B, 2012, 85, 155208.
${ }^{61}$ A. Jain, G. Hautier, C. J. Moore, S. P. Ong, C. C. Fischer, T. Mueller, K. A. Persson and G. Ceder, Comput. Mater. Sci., $2011, \mathbf{5 0}, 2295-2310$.

${ }^{62}$ A. J. Toumar, S. P. Ong, W. D. Richards, S. Dacek and G. Ceder, Phys. Rev. Applied, 2015, 4, 064002.

${ }^{63}$ J. Harl and G. Kresse, Phys. Rev. Lett., 2009, 103, 056401.

${ }^{64}$ M. Kim, W. J. Kim, E. K. Lee, S. Lebègue and H. Kim, Int. J. Quantum Chem., 2016, 116, 598-607.

${ }^{65}$ T. Björkman, A. Gulans, A. V. Krasheninnikov and R. M. Nieminen, Phys. Rev. Lett., 2012, 108, 235502.

${ }^{66}$ F. Zhou, M. Cococcioni, C. A. Marianetti, D. Morgan and G. Ceder, Phys. Rev. B, 2004, 70, 235121.

${ }^{67}$ D.-H. Seo, A. Urban and G. Ceder, Phys. Rev. B, 2015, 92, 115118.

${ }^{68}$ J. P. Allen and G. W. Watson, Phys. Chem. Chem. Phys., 2014, 16, 21016-21031.

${ }^{69}$ B. J. Morgan and P. A. Madden, Phys. Rev. B, 2012, 86, 035147. 